\title{
CONTINENCE CARE
}

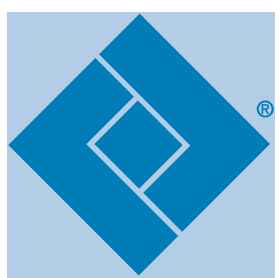

\section{Multicenter Cohort Study to Assess the Impact of a Silver-Alloy and Hydrogel-Coated Urinary Catheter on Symptomatic Catheter-Associated Urinary Tract Infections}

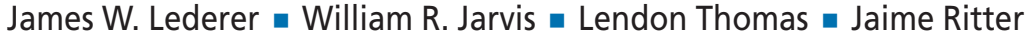

\section{ABSTRACT}

PURPOSE: The purpose of this study was to determine the effect of a silver-alloy hydrogel catheter on symptomatic catheter-associated urinary tract infections (CAUTIs). DESIGN: Multicenter before-after non-randomized cohort study.

SUBJECTS AND SETTING: Seven acute care hospitals ranging in size from 124 to 607 beds participated in this study. The study population included adult patients with a positive urine culture 2 or more days after admission, who underwent Foley catheterization.

METHODS: Catheter-associated urinary tract infection surveillance was conducted at each hospital for at least 3 months during the use of a standard catheter and 3 months during the use of the silver-alloy hydrogel catheter. Both the National Healthcare Safety Network (NHSN) surveillance and a clinical definition of CAUTI were used for rate calculation.

RESULTS: A 47\% relative reduction in the CAUTI rate was observed with the silver-alloy hydrogel catheter compared to the standard catheter when both infection definitions were used $(0.945 / 1000$ patient days vs $0.498 / 1000$ patient days) (odds ratio $=0.53 ; P<.0001$; $95 \% \mathrm{Cl}: 0.45-0.62)$. When only NHSN-defined CAUTIs were considered, a $58 \%$ relative reduction occurred in the silver-alloy hydrogel period $(0.60 / 1000$ patient days vs $0.25 / 1000$ patient days) (odds ratio $=0.42 ; P<$ $.0001 ; 95 \% \mathrm{Cl}: 0.34-0.53)$. Antimicrobial days for CAUTIs decreased from 1165 (standard catheter period) to 406 (silver-alloy hydrogel period).

CONCLUSIONS: Use of a silver-alloy hydrogel urinary catheter reduced symptomatic CAUTI occurrences as defined by both NHSN and clinical criteria.

KEY WORDS: catheter-associated urinary tract infection, Foley catheter, silver alloy catheter, silver catheter, urinary tract infection.

\section{Introduction}

Urinary tract infections (UTIs) are one of the most common healthcare-associated infections; approximately $80 \%$ are related to the use of indwelling urinary catheters. ${ }^{1,2}$ Healthcare-associated bacteriuria or funguria develops in up to $25 \%$ of catheterized patients with a portion of those progressing to symptomatic infection. ${ }^{2}$ Catheterassociated urinary tract infection (CAUTI), and to a lesser extent bacteriuria, is associated with morbidity, mortality, and excess healthcare costs. ${ }^{1-4}$ Cost estimates vary, but a case-control study suggested that patients with CAUTI had excess costs of $\$ 3803$ compared to patients without infection. ${ }^{1}$

Patient risk factors for CAUTIs have been identified; they include older age, female gender, diabetes mellitus, and duration of catheterization. ${ }^{1,5}$ Additional risk factors are specifically associated with the indwelling urinary

- James W. Lederer, MD, Vice President, Clinical Improvement at Novant Health, Winston-Salem, North Carolina.

- William R. Jarvis, MD, President, Jason and Jarvis Associates, LLC, Hilton Head Island, South Carolina.

- Lendon Thomas, BS, CIC, Clinical Specialist C. R. Bard. Inc., Bard Medical Division, Covington, Georgia.

- Jaime Ritter, MPH, CIC, Senior Manager C. R. Bard, Inc, Bard Medical Division, Covington, Georgia.

Lendon Thomas and Jaime Ritter are employees of, and William R. Jarvis is a consultant for C. R. Bard, Inc., Bard Medical Division. James W. Lederer has nothing to disclose.

This is an open-access article distributed under the terms of the Creative Commons Attribution-NonCommercial-NoDerivitives 3.0 License, where it is permissible to download and share the work provided it is properly cited. The work cannot be changed in any way or used commercially.

Correspondence: Jaime Ritter, MPH, CIC, C. R. Bard, Inc. Bard Medical Division, 8195 Industrial Blvd, Covington, GA 30014 (jaime.ritter@crbard.com).

DOI: 10.1097/WON.0000000000000056 
catheter system and include contamination of the catheter during insertion or while indwelling and contamination of the drainage system.1,2 Multiple strategies and techniques have been studied to reduce the risk of CAUTI, many of which have become standard practice for catheterized patients (eg, sterile insertion and closed drainage systems). ${ }^{1,2}$ Urinary catheter-coating technologies have been introduced to prevent the development of biofilm on the catheter surface (both internal and external surfaces). Possibly the most well-studied technology, the silver-alloy hydrogel (SAH) indwelling catheter, has been demonstrated in multiple large clinical trials and smaller case studies to reduce the incidence of CAUTI compared to non-silver-coated catheters. ${ }^{5,6}$ Published studies on CAUTI prevention strategies, including SAH catheters, have examined a variety of endpoints, including bacteriuria and surveillance-defined UTI. ${ }^{3,4,7}$

Until January 2009, the Centers for Disease Control and Prevention's (CDC's) National Healthcare Safety Network (NHSN) included both symptomatic urinary tract infection (SUTI) and asymptomatic bacteriuria (ASB) in their definition of CAUTI. ${ }^{8}$ Subsequently, the CDC revised its NHSN surveillance definition of CAUTI by modifying the criteria for symptomatic infection, adding a category and definition for asymptomatic bacteremic urinary tract infection, and removing ASB completely. ${ }^{9}$ Because of the change in NHSN definition, much of the previously published evidence on CAUTI prevention strategies has been called into question, because many combined ASB and SUTI as the endpoint. Specifically, some clinicians have suggested that studies examining either ASB or a combination of SUTI and ASB may not be as relevant as studies examining SUTI alone. ${ }^{5}$

It is important to note that clinical CAUTI has not been clearly or systematically defined and may be different than the more widely quoted CDC NHSN surveillance definitions. Epidemiologic surveillance definitions are designed for consistency of interpretation and thus have very strict parameters in order to meet the surveillance case definition. ${ }^{10}$ The clinical definition of CAUTI appears to be more subjective. Diagnosis and treatment are based on daily observations of patient progress (improvement or deterioration) and local signs and symptoms such as fever, change in mental status, change in character of the urine, pyuria, leukocytosis, etc. As a result, many patients who have clinical UTI by observation and who are managed and treated as infected may not meet the strict CDC NHSN surveillance definition for CAUTI.

The principal aim of this study was to determine the CAUTI rate before and after introduction of the SAH indwelling urinary catheter system. The analysis compared CAUTI rates between standard non-silver-coated (STD) catheters and SAH catheters using the revised 2009 CDC NHSN criteria and a combined measure of the CDC's NHSN and clinically defined CAUTI (Figures 1 and 2).

\section{Methods}

This retrospective, multicenter, before-after study was designed to compare CAUTI rates between patients receiving standard, non-silver-coated urinary catheters and patients receiving silver-alloy and hydrogel-coated Foley catheters using several CAUTI definitions. Data were collected from 7 hospitals, which had previously used STD urinary catheters, and then switched to the SAH catheters (Bardex I.C. Foley Catheter, C.R. Bard, Inc, Covington, Georgia). The most commonly used standard catheter was a hydrogel-coated latex catheter; noncoated silicone catheters were generally used only if a patient was allergic to latex. Most catheters were provided in a preconnected tray system (materials necessary for aseptic insertion; catheter attached to either a drainage bag or urine meter). Hospitals were located in the Eastern and Midwestern regions of the United States. The Institutional Review Board at each participating hospital reviewed and approved the study protocol prior to initiation of any study-related activities.

Adult patients aged between 18 and 89 years with a significant positive urine culture (NHSN criteria of $\geq 10^{3}$ colony-form unit per milliliter with no more than 2 species of microorganisms) 2 or more calendar days after hospital admission, who received an indwelling urinary catheter during the study time periods were included in the study. Patients with urinary diversions, certain preexisting conditions affecting the bladder (eg, active bladder cancer, chronic indwelling urinary catheter in place), or those undergoing an invasive urologic surgery were excluded.

In order to capture house-wide, hospital-inserted CAUTIs, all positive urine cultures ( $\geq 1000$ colony-forming units per milliliter with no more than 2 species of microorganisms) occurring in a minimum of 3 consecutive months were screened in each time period. It was expected that approximately 3 consecutive months of screened urine culture data would yield at least 50 candidates generally representing the hospital's patient population. However, the time frame was extended for small hospitals to allow for the collection of an adequate sample size. Initial screening of the positive urine culture list allowed for exclusion of patients based on age and time from hospital admission to culture. Medical records of all candidates meeting the initial screening criteria were further reviewed to determine if all inclusion and exclusion criteria were met.

At the end of each study time period data were retrospectively collected by a team of experienced infection preventionists, all certified in infection control by the Certification Board of Infection Control and Epidemiology, Inc. Each candidate's medical record was reviewed to gather pertinent information including demographic data (eg, age, gender, length of stay), urinary catheterization data (eg, length of catheterization, day of catheter insertion), 
Symptomatic Urinary Tract Infection (SUTI)

Patient had an indwelling urinary catheter at the time of, or discontinued within 48 hours prior to, specimen collection or onset of signs or symptoms

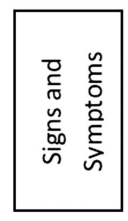

$$
\begin{aligned}
& \text { At least } 1 \text { of the following with no other recognized cause: } \\
& \text { - Fever - Suprapubic tenderness }
\end{aligned}
$$
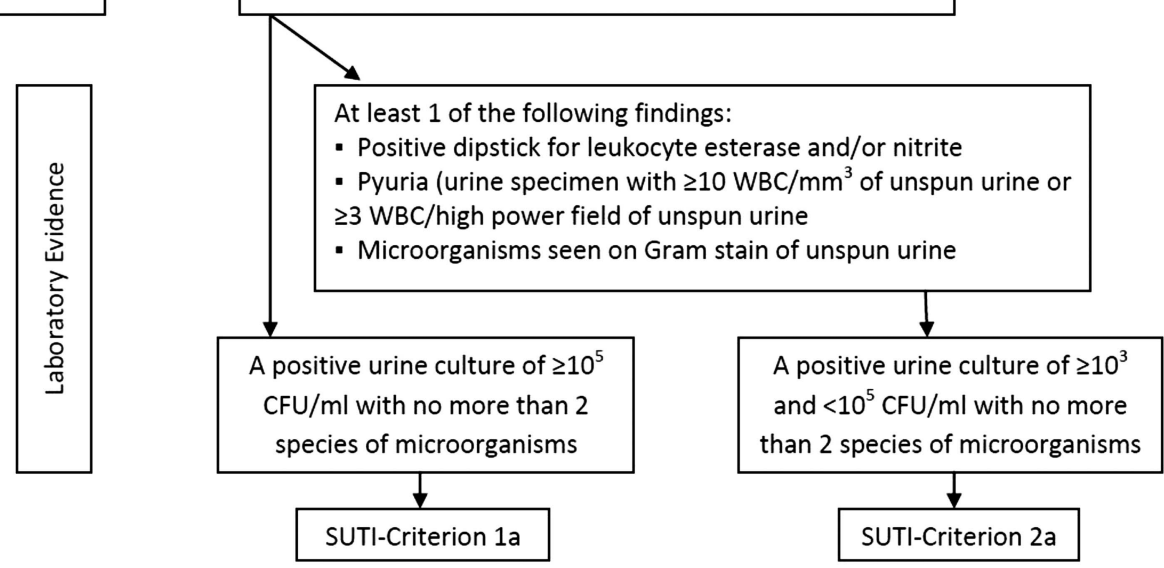

Asymptomatic Bacteremic Urinary Tract Infection (ABUTI)

Patient had an indwelling urinary catheter at the time of, or discontinued within 48 hours prior to, specimen collection
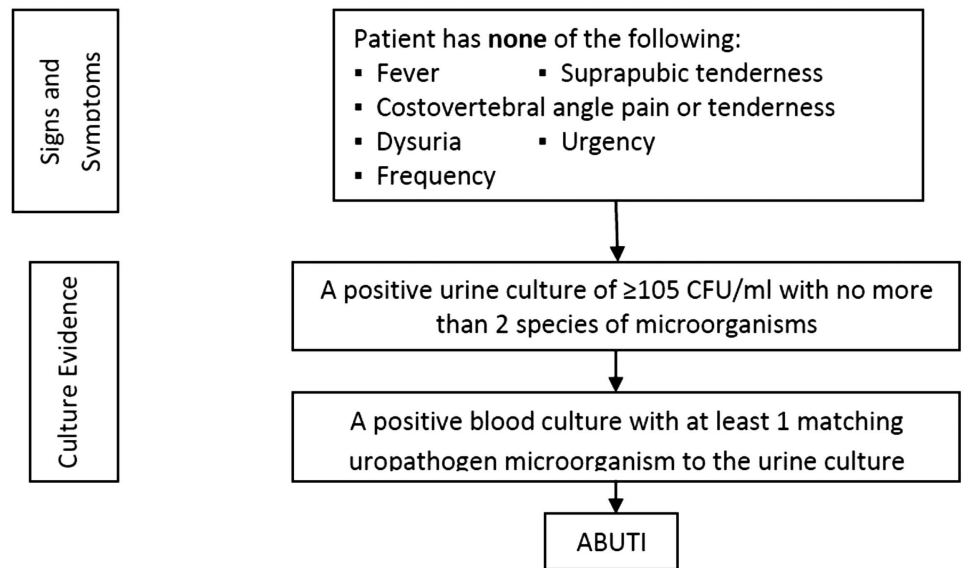

FIGURE 1. Centers for Disease Control and Prevention's National Healthcare Safety Network catheter-associated urinary tract infection definitions. WBC indicates white blood cell.

urine culture data, urinalysis results, signs and symptoms of CAUTI, and physician diagnosis and treatment. Collected data were reviewed to determine if CDC NHSN or our clinically defined CAUTI (CLIN-CAUTI) definition criteria were met. Each data set was audited by one of the team infection preventionists to ensure the consistency of data collection and application of CAUTI definitions (infection preventionists were not allowed to audit their own work).

Ideally, CAUTI rates are calculated using indwelling urinary catheter utilization days. While catheter utilization days often are available for intensive care units, they are rarely available house-wide. Because of this, adult, acute care patient-days were used as the denominator for CAUTI rate calculations.

\section{Data Analysis}

Based on the assumption of a NHSN-CAUTI/CLIN-CAUTI rate of 1.4 per 1000 patient days in the STD catheter group and 0.98 per 1000 patient days in the SAH group, a 2-sided alpha of .05 and power of $80 \%, 485$ qualified cultures per study group were required. If only NHSN-CAUTIs were included in rate calculations, an estimated 552 qualified cultures per study group were required. All data were 
Clinical Catheter-Associated Urinary Tract Infection (CLIN-CAUTI)

Patient had an indwelling urinary catheter at the time of, or discontinued within

48 ho prior to, specimen collection or onset of signs or symptoms

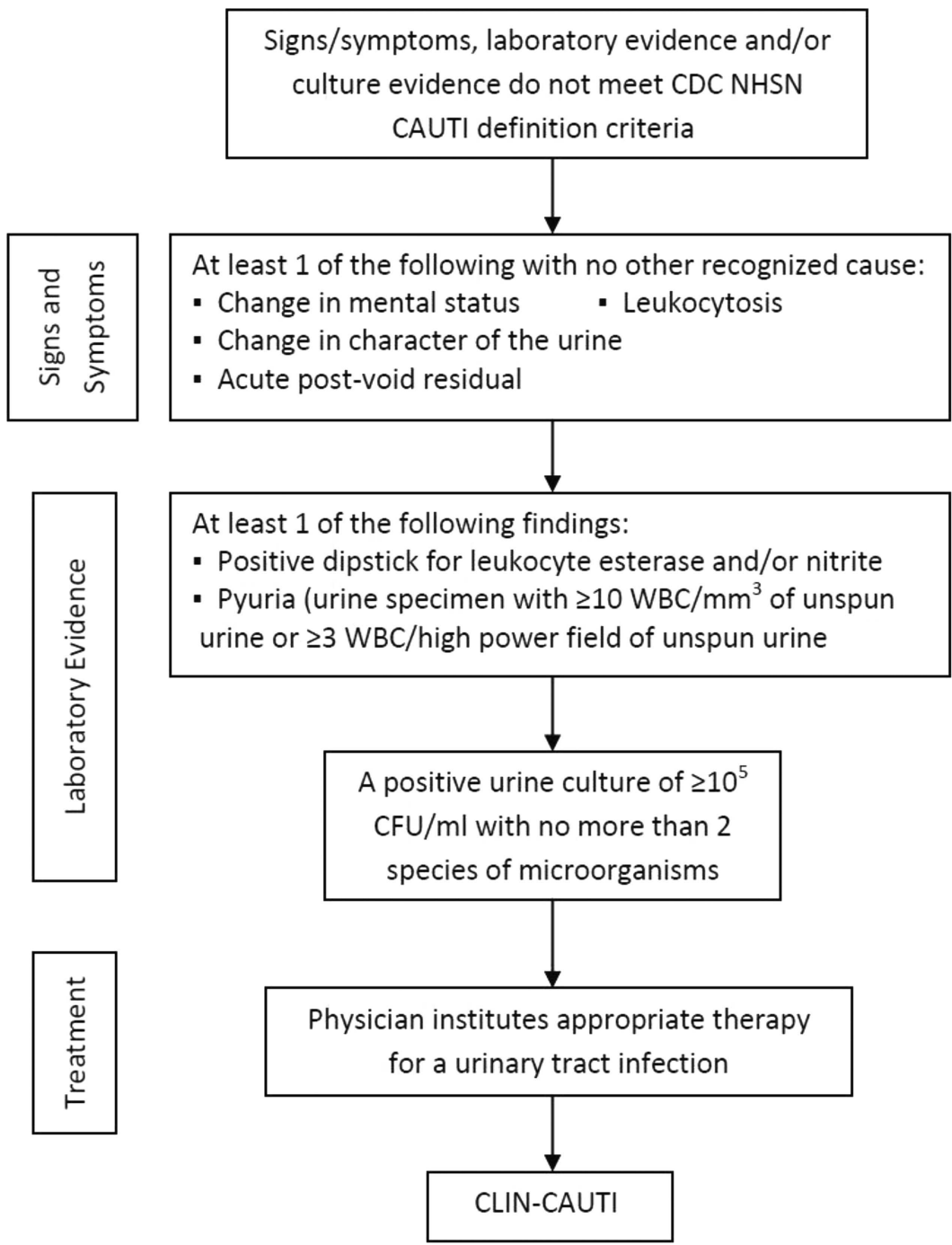

FIGURE 2. Clinically infected catheter-associated urinary tract infection definition. CAUTI indicates catheter-associated urinary tract infection; CDC, Centers for Disease Control and Prevention; CFU, colony-forming unit; NHSN, National Healthcare Safety Network.

collected and managed in a Microsoft Access database (Microsoft, Redmond, Washington) and analyzed in SAS Analytics (SAS Institute, Inc, Cary, North Carolina). The Wilcoxon rank sum test was used to analyze continuous variables and the Fisher exact test was used for categorical variables.

\section{Results}

Seven hospitals, representing 2778 active acute care beds, participated in the study. Six of the facilities were community hospitals ranging in size from 124 to 607 beds (median $=289$ ); the seventh facility was a teaching hospital with 921 beds. There were no significant changes in indwelling urinary catheter practices or overall catheter usage between the 2 data collection time periods at any of the hospitals. A total of 64 months of data were collected (32 in each time period), with each hospital contributing data for at least 6 months (range, 6-14 months).

Eight hundred six candidates (854 cultures) were identified in the STD period after initial screening and 774 candidates (801 cultures) identified in the SAH period. Medical record review identified 453 qualified patients in the STD time period meeting all inclusion and exclusion criteria (501 cultures) with 400 qualified patients ( 427 cultures) identified in the SAH time period (Table 1). 
TABLE 1.

\section{Patient and Medical Record Screening}

\begin{tabular}{lll}
\hline & \multicolumn{1}{c}{$\begin{array}{c}\text { Standard } \\
\text { Catheter }\end{array}$} & \multicolumn{1}{c}{$\begin{array}{c}\text { Silver-Alloyl } \\
\text { Hydrogel Catheter }\end{array}$} \\
\hline $\begin{array}{l}\text { Total patient candidates } \\
\text { Total qualified patients }\end{array}$ & 806 (854 cultures) & 774 (801 cultures) \\
$\begin{array}{l}\text { Total disqualified } \\
\text { patients }\end{array}$ & $353(401$ cultures) & 400 (427 cultures) \\
$\begin{array}{l}\text { Preexisting condition } \\
\text { a }\end{array}$ & $38(10.8 \%)$ & $374(48.3 \%)$ \\
\hline $\begin{array}{l}\text { No study catheter } \\
\text { Record not available }\end{array}$ & $303(85.8 \%)$ & $41(11.0 \%)$ \\
\hline Reco & $13.4 \%)$ & $22(5.9 \%)$ \\
\hline
\end{tabular}

apreexisting condition: active bladder cancer, chronic hemodialysis, invasive urologic procedure, urinary diversion.

${ }^{b}$ No study catheter: admitted with Foley catheter in place, Foley catheter inserted outside of study time period, no Foley catheter inserted throughout admission.

One hundred seventy-four CAUTIs occurred in 170 patients in the STD period; 90 CAUTIs occurred in 88 patients in the SAH time period. A 47\% relative reduction in the CAUTI rate was observed with the use of the SAH catheter compared to the STD catheter (0.945 per 1000 patient days vs 0.498 per 1000 patient days) (odds ratio $=$ $0.527 ; P<.0001 ; 95 \%$ CI: $0.445-0.623)$. Of the total CAUTIs identified, 111 (64\%) in the STD period and 46 (51\%) in the SAH period met CDC NHSN CAUTI definition. This resulted in a $58 \%$ relative reduction in the NHSN CAUTI rate in the SAH period compared to the STD period (0.603 per 1000 patient days vs 0.254 per 1000 patient days) (odds ratio $=0.42 ; P<.0001 ; 95 \%$ CI: 0.34-0.53) (Figure 3). Of the CDC NHSN-defined CAUTIs, 2 asymptomatic bacteremic urinary tract infections occurred in patients with the STD catheter and 1 occurred in a patient with the SAH catheter. Among patients with CAUTI, 5 secondary bacteremias were identified in the STD period while none occurred in the SAH period.

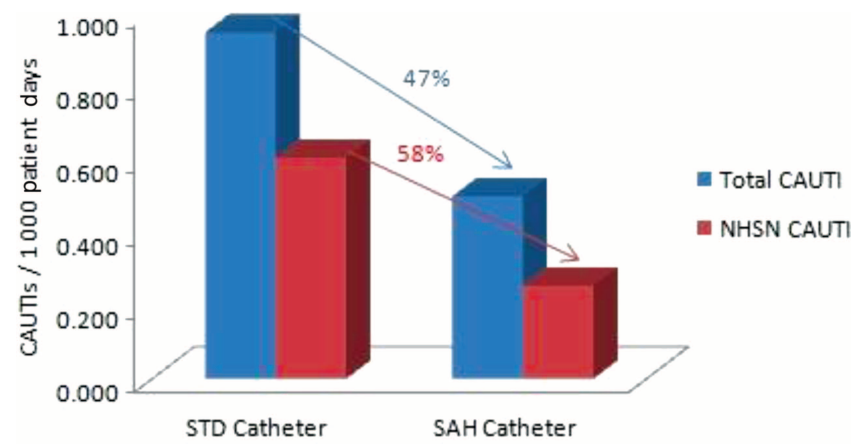

FIGURE 3. Catheter-associated urinary tract infection rates. NHSN indicates National Healthcare Safety Network.

Patients with CAUTIs were predominately female, older than 65 years, and had an average length of stay of more than 12 days. Most CAUTIs occurred in patients admitted with medical diagnoses (73\%) who had an indwelling urinary catheter in place for more than 3 days. There were no significant differences in gender, age, length of stay, or length of catheterization in CAUTI patients between the 2 time periods. Length of stay and length of catheterization appeared to be shorter for qualified non-CAUTI patients although this was not statistically tested (Table 2).

Four main areas of the hospital where indwelling urinary catheters were inserted were associated with CAUTIs: for STD catheters, $46 \%$ were inserted in the emergency department, $17 \%$ in medical/surgical units, $17 \%$ in critical care units, and $16 \%$ in the operating room. For SAH catheters, $32 \%$ were inserted in the emergency department, $30 \%$ in medical/surgical units, $20 \%$ in the operating room, and $12 \%$ in critical units. The mean number of days from catheter insertion to onset of infection in the STD period was 8.6 days (median 6 days, range 1-59 days) and 7.7 days (median 5 days, range 1-53 days) in the SAH period. Nevertheless, most infections occurred within the first 10 days of catheterization for both groups (Table 3 ).

\section{TABLE 2.}

\section{Patient Demographic Information}

\begin{tabular}{lccc}
\hline & Standard Catheter & Silver-Alloy/Hydrogel Catheter & $P$ \\
\hline Gender & & & .44 \\
$\quad$ Female & $75 \%$ & $80 \%$ & \\
$\quad$ Male & $25 \%$ & $20 \%$ & .22 \\
Mean age (median) & 66.7 years (70) & 68.8 years (70.5) & .67 \\
Mean LOS (median) in CAUTI patients & 19.6 days (13) & $16.2(14)$ & .32 \\
Mean LOC (median) in CAUTI patients & 14.8 days (8.5) & $10.9(8)$ & .60 \\
Mean LOS (median) in Non-CAUTI patients & 15.7 days (11) & $14.1(10)$ & .05 \\
Mean LOS (median) in Non-CAUTI patients & 10.8 days (6) & 9.2 days (6) & \\
\hline
\end{tabular}

Abbreviations: CAUTI, catheter-associated urinary tract infection; LOC, length of catheterization; LOS, length of stay. 
TABLE 3.

\begin{tabular}{lcc}
\hline $\begin{array}{l}\text { Catheterization Day of CAUTI Onset } \\
\text { Day Group of } \\
\text { CAUTI Onset }\end{array}$ & $\begin{array}{c}\text { Standard } \\
\text { Catheter, \% }\end{array}$ & $\begin{array}{c}\text { Silver-Alloy/Hydrogel } \\
\text { Catheter, \% }\end{array}$ \\
\hline Days 1-2 & 23 & 14 \\
Days 3-5 & 25 & 38 \\
Days 6-10 & 26 & 24 \\
Days $\geq 11$ & 26 & 24 \\
\hline
\end{tabular}

Abbreviation: CAUTI, catheter-associated urinary tract infection.

Approximately $68 \%$ of all CAUTIs in the STD group and $58 \%$ in the SAH group were attributed to gram-negative organisms, the most common being Escherichia coli, Klebsiella pneumoniae, and Pseudomonas aeruginosa. Grampositive organisms were recovered in $19 \%$ and $22 \%$ of the CAUTIs in the STD and SAH cohorts, respectively. The most common gram-positive organisms were Enterococcus spp, Staphylococcus aureus, and coagulase-negative Staphylococcus spp. Yeast, primarily Candida albicans and other Candida spp, were recovered from $14 \%$ of the infections in the STD period and $20 \%$ in the SAH period.

By definition, all of the patients with CLIN-CAUTIs were treated with antimicrobials; however, $92 \%$ and $78 \%$ of the CDC NHSN-CAUTIs were treated in the STD and SAH time periods, respectively. Among all CAUTI patients, there were 1165 antimicrobial treatment days in the STD cohort (630 intravenous therapy days; 535 oral therapy days) and 406 treatment days in the SAH cohort (206 intravenous therapy days; 200 oral therapy days). The mean antimicrobial treatment days for CAUTI patients were 6.85 (median 6.0) in the STD group and 4.61 (median $4.0)$ in the SAH group $(P=.007)$.

\section{Discussion}

After the advent of closed drainage systems in the 1960s, little research focused on developing new CAUTI prevention strategies or technologies until the last decade of the 20th century. In the early 1990s, different types of catheter coatings were introduced and studied in an attempt to reduce these infections. Although there were mixed results for some coating technologies (eg, silveroxide ${ }^{11}$ ), the SAH catheter used in our study was supported by multiple studies. ${ }^{3,12,13}$ Because of the change in CDC NHSN definitions in 2009, there are very few studies that provide solid evidence of the effectiveness of any CAUTI prevention strategies or technologies, including the $\mathrm{SAH}$ catheter evaluated in our study. A randomized study examining the effect of 2 antimicrobial catheters was published in 2012. That study focused on surgical patients and the definition of symptomatic UTI used was based primarily on patient diaries (collected up to 6 weeks postcatheterization) and physician treatment rather than previous or current CDC NHSN criteria. ${ }^{7}$

Current CAUTI prevention strategies focus on limiting exposure to indwelling urinary catheters (ie, limiting use, decreasing catheter days); however, our data examining catheter days and time from catheterization to onset of infection appear to suggest that there is still a fairly significant risk of infection in patients who are catheterized for even a short period of time ( $\leq 3$ days). Additionally, for patients who require the use of an indwelling urinary catheter for a longer period of time, the professional literature reveals few if any new preventive interventions on the horizon. ${ }^{5}$ Based on their ongoing efforts to reduce the rate of CAUTI within their facilities, the 7 hospitals participating in this study opted to evaluate the effectiveness of the SAH catheter. With the significant reduction seen in the CAUTI rates between the STD and SAH time periods in hospitals already implementing comprehensive prevention programs, it appears that the addition of the SAH catheter offers a complimentary effect at a minimum.

We elected to assess the incidence of infection based on not only the new 2009 CDC NHSN CAUTI definition, but also a "clinical" definition, and intended to reproduce what clinicians actually do when managing patients with clinical signs and symptoms indicative of probable CAUTI. Our goal was to ensure that we captured as many clinically relevant infections as possible. Recognizing that there could be a variety of clinical indicators of CAUTI that are not currently included in the CDC NHSN definition (eg, change in mental status and leukocytosis), we opted to include several in our CLIN-CAUTI definition. To strengthen the definition, we required microbiologic and microscopic evidence of CAUTI as well as documented treatment. Interestingly, when we examined the overall reduction in total CAUTIs (including the CDC NHSN and our CLIN-CAUTI), the reduction was $47 \%$ while the reduction was 58\% when we included only the new CDC NHSNdefined CAUTIs. These data would seem to suggest that the SAH catheter may be most effective against symptomatic CAUTI. Additionally, our findings suggest that the definition of CAUTI used to study prevention technologies can have a significant impact on the observed impact of any intervention being assessed.

Because CAUTIs have been known as a breeding ground for antimicrobial-resistant microorganisms, ${ }^{2}$ we collected data on all CAUTIs receiving treatment. The vast majority, but not all of the CAUTIs, were treated in both time periods. There were 1165 total antimicrobial days in the STD time period. Given the $47 \%$ relative reduction in CAUTIs, one could assume that we would see a similar trend in antimicrobial days (an estimated 617 days in the SAH time period); however, we actually observed only 406 antimicrobial days (a 65\% relative reduction). It is unclear why antimicrobial days were lower in the SAH period than expected, but it could be related to a slightly delayed onset of CAUTI and/or a 
change in symptomatology or perceived severity of infection in the SAH time period.

\section{Limitations}

There are several limitations of our study. First, we conducted a before-after cohort study. While a randomized, controlled trial may have yielded more robust evidence, our study represents the typical, everyday process an individual hospital uses to evaluate the effectiveness of infection-prevention strategies. Furthermore, a recent randomized controlled study of impregnated urinary catheters focused primarily on surgical patients, accepted symptomatology for up to 6 weeks after urinary catheterization, and failed to use either the old or new CDC NHSN definition of CAUTI. ${ }^{7}$ Our study was conducted hospitalwide and therefore captured all microbiological confirmed CAUTIs, not just those occurring in intensive care units, which is customary in most hospitals performing CDC NHSN device-associated infection surveillance. In addition, because data were collected retrospectively, we were limited to outcomes that were documented in the medical record and had to assume that if something was not documented, it in fact did not occur. Poor documentation practice regarding indwelling urinary catheter insertion and care and potential CAUTI symptoms may have occurred in some study sites. However, the volume of medical records reviewed and multiple study sites may have reduced the likelihood of this possible bias.

We used positive urine cultures to identify candidate patients instead of indwelling urinary catheterization records. Thus, all patients with a positive culture urine were evaluated for inclusion. Unfortunately, even with the advent of electronic medical records, it is difficult to identify patients who receive an indwelling catheter during their hospital course. Although using positive cultures to identify candidate patients may not have been ideal, because CAUTI definitions require a positive culture, it is unlikely that infections went undetected in our study. Lastly, we used patient-days to calculate CAUTI infection rates rather than device days. Again, even with the advent of electronic medical records, it remains difficult to capture house-wide catheter days. While hospitals performing CDC NHSN CAUTI surveillance have developed systems to capture catheter days in their intensive care units, few have the ability to capture the data in other areas of the hospital. Kim and colleagues ${ }^{14}$ conducted a study of 99,834 patients over a 2-year period (2002-2004) comparing CAUTI rates using both device-days and patient-days as the denominator in their calculations and found that the 2 rates were highly correlated. They concluded that rates based on patient-days can serve as a surrogate for rates based on device-days. In addition, the use of indwelling urinary catheters was similar in all time periods. It is, therefore, unlikely that the relative reduction in CAUTI rates between the 2 study time periods would be significantly impacted by the use of a different denominator for rate calculation.

\section{Conclusions}

Results of this study demonstrated that the SAH Foley catheter was associated with a significant reduction in CAUTIs rates in a real-world setting. The effect was greater in CDC NHSN-defined symptomatic infections than in other clinically defined CAUTIs, although SAH Foley catheters were associated with decreased CAUTI rates regardless of which CAUTI definition was used.

\section{ACKNOWLEDGMENT}

This study was sponsored by C. R. Bard, Inc., Bard Medical Division. Participating institutions: Forsyth Medical Center, Winston-Salem, North Carolina; Marion Regional Hospital, Marion, South Carolina; Memorial Hospital of Martinsville, Martinsville, Virginia; Middlesex Hospital, Middletown, Connecticut; North Memorial Medical Center, Robbinsdale, Minnesota; Park Nicollet Institute, St. Louis Park, Minnesota; and Presbyterian Hospital, Charlotte, North Carolina.

\section{References}

1. Salgado CD, Karchmer TB, Farr BM. Prevention of catheterassociated urinary tract infection. In: Prevention and Control of Nosocomial Infections. Philadelphia, PA: Lippincott Williams \& Wilkins; 2003:297-311.

2. Maki DG, Tambyah PA. Engineering out the risk for infection with urinary catheters. Emerg Infect Dis. 2001;7:342-347.

3. Karchmer TB, Giannetta ET, Muto CA, et al. A randomized crossover study of silver-coated urinary catheters in hospitalized patients. Arch Int Med. 2000;160:3294-3298.

4. Johnson JR, Kuskowski MA, Wilt TJ. Systematic review: antimicrobial urinary catheters to prevent catheter-associated urinary tract infection in hospitalized patients. Ann Int Med. 2006; 144:116-126.

5. HICPAC. Guidelines for Prevention of Catheter-Associated Urinary Tract Infection. Atlanta, GA: Centers for Disease Control and Prevention; 2009.

6. Schumm K, Lam TBL. Types of urethral catheters for management of short-term voiding problems in hospitalized adults. In: The Cochrane Collaboration. Chichester, England: The Cochrane Library; 2008:(3) Wiley.

7. Pickard R, Lam T, MacLennan G, et al. Antimicrobial catheters for the reduction of symptomatic urinary tract infection in adults requiring short-term catheterization in hospital: a multicentre randomized controlled trial. Lancet. 2012;380 (9857):1927-1935.

8. Division of Healthcare Quality Promotion, Centers for Disease Control and Prevention. The National Healthcare Safety Network (NHSN) Manual. Atlanta, GA: Centers for Disease Control and Prevention; 2008.

9. Division of Healthcare Quality Promotion, Centers for Disease Control and Prevention. The National Healthcare Safety Network (NHSN) Manual. Atlanta, GA: Centers for Disease Control and Prevention; 2009.

10. Arias KM. Surveillance. In: APIC Text of Infection Control and Epidemiology. Washington, DC: Association for Professionals in Infection Control and Epidemiology, Inc.; 2005:6-7.

11. Riley DK, Classen DC, Stevens LE, Burke JP. A large randomized clinical trial of a silver-impregnated urinary catheter: lack of 
efficacy and staphylococcal super-infection. Am J Med. 1995; 98:349-356.

12. Maki DG, Knasinski V, Halvorson K, Tambyah PA. A novel silver-hydrogel-impregnated indwelling catheter reduces CAUTIs: a prospective double-blind trial [abstract]. Infect Control Hosp Epidemiol. 1998;19:682.
13. Rupp ME, Fitzgerald T, Marion N, et al. Effect of silver-coated urinary catheters: efficacy, cost-effectiveness, and antimicrobial resistance. Am J Infect Control. 2004;32:445-450.

14. Kim AT, Kirchner CD, Fishman DN, Dhond AJ, Abrutyn E. Measuring device days vs. patient days for patients with urinary catheters [abstract]. Am J Infect Control. 2005;33:e69.

\section{Call for Authors: Continence Care}

Original research, case studies and case series addressing, in particular:

- Urinary or fecal stream diversion: indwelling urethral, suprapubic catheters or fecal/bowel management system.

- Pelvic floor muscle rehabilitation protocols for stress, urge and mixed urinary incontinence in men or women.

- Current state of the science presented in systematic reviews and/or meta analyses.

- Evidence based management of incontinence associated dermatitis or moisture associated skin damage.

- Incidence and prevalence of incontinence in understudied populations.

- Quality of life issues associated within continence, care-giving, catheter management, prevention of incontinence. 